\title{
Optimization of Hybrid Electric Bus Control Strategy with Hybrid Optimization Algorithm
}

\author{
Gao Jianping ${ }^{1, a}$, Liu Zhennan ${ }^{1, b}$, Guo Zhijun ${ }^{1, c}$, Wei Yuehui ${ }^{1, d}$ \\ ${ }^{1}$ Vehicle \& Motive Power Engineering College, Henan University of Science \& Technology, \\ Luoyang 471003, China \\ agjpcar@gmail.com, ${ }^{\mathrm{b}}$ liukhev@163.com, ${ }^{\mathrm{C}}$ gzhj1970@163.com, ${ }^{\mathrm{d}}$ weigaosun@163.com
}

Keywords: Hybrid Electric Bus, control strategy, multi-island genetic algorithm, hybrid optimization algorithm

\begin{abstract}
Hybrid Electric Bus (HEB) and directly influences the dynamic performance and fuel economy. For achieving the best fuel economy and keeping the battery for a long time, First, power analytic control strategy was built; then, the hybrid optimization algorithm (HOA) based on Multi-island genetic Algorithm (MIGA) and NLPQL was built by ISIGHT software. HOA is adopted in control strategy parameters of HEB optimization. The results show that the best result can be obtained in few iterative times by HOA, the calculation time was reduce by 12 hours, the fuel economy was improved by $12 \%$ and find the rules between control strategy parameters and fuel economy 、 the balance of the battery state of charge(SOC).
\end{abstract}

\section{Introduction}

The core and base of Hybrid electric Bus (HEB) is multi-energy powertrain control system. The objective of the HEB's control strategy reduce fuel consumption 、 exhaust emission and keep the battery for a long time to save when the automobile remains its original dynamics ${ }^{[1-3]}$. As core of power distribution directly, the control strategy affects the dynamic, economic of the vehicle. So it is important to research the optimization of control strategy in theoretical study and application.

It is transferred the multi objective problem to single objective problem using the method of weighting, its pareto optimal solution was obtained with genetic algorithm, but Premature convergence has been a main problem in genetic algorithms, which converges on local optimum not global optimal solution ${ }^{[4-6]}$. A software which combines genetic algorithm with traditional gradient algorithm SQP is put forward and it makes the best use of the ability of the global search ability of genetic algorithm and gradient algorithm fast convergence. But it use ADVISOR, which is largely different from the actual situation to get the imprecise result ${ }^{[7-8]}$. According to combine MATLAB and AVL Cruise, an genetic algorithm to optimize control strategy was adopted, but it not only needs write genetic algorithm by hand, but also takes long time to optimize ${ }^{[9]}$.

In order to solve the problems occurred during the control strategy optimization, the hybrid optimization algorithm with composing of MIGA and NLPQL is built by SIGHT software platform .After global exploration by MIGA get the optimum areas, and then NLPQL come into optimum areas to get the local optimization. This optimization algorithm is used to optimal control strategy of HEB to get the best fuel economy and keep the balance of battery SOC.

\section{HEB simulation analysis model and control strategy}

Simulation analysis model is a powerful tool to study HEB control strategy. As a forward simulation software, compared with reverse simulation software, AVL Cruise not only realize dynamic calculation ,but also have advantages of the higher calculation accuracy and realistic .So it is fit to the research of control strategy. 


\subsection{HEB simulation analysis model based on AVL Cruise}

HEB uses a typical dual-clutch and Single-axle Parallel configuration show as fig.1. It includes an engine, battery,a motor and AMT. Engine can work independently to supply the power. The driving motor is not only driving unit but also a system being used starting engine and generating electricity (ISG).the battery connect the motor, which can keep the balance of the charging and discharging based on control strategy. Some key parameters of HEB as show in Table 1.

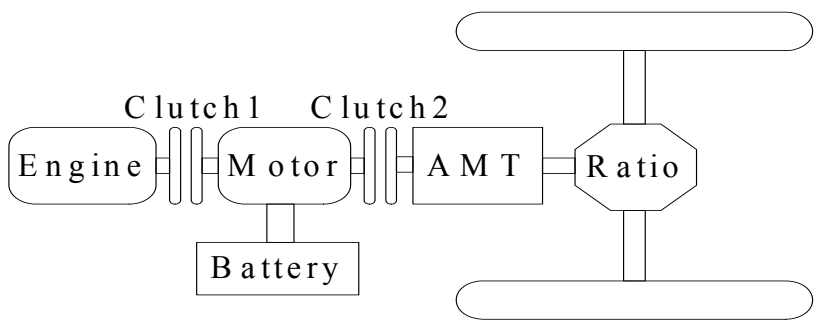

Figure 1. Structure of HEB

Table 1 . Several key parameters of HEB

\begin{tabular}{ccc}
\hline & Parameter & Type and Value \\
\hline \multirow{3}{*}{ Engine } & Type & 4-cylinder diesel engine \\
& Max Power[kw] & 132 \\
& Max Torque(Speed) $\left[\mathrm{kw} / \mathrm{r} \cdot \mathrm{min}^{-1}\right]$ & $655(250)$ \\
\hline \multirow{3}{*}{ Battery } & Type & lithium battery \\
& Capacity $[\mathrm{Ah}]$ & 70 \\
\hline \multirow{2}{*}{ Motor } & Rated voltage[V] & 358 \\
& Type & Ac induction motor \\
& Rated power $[\mathrm{kw}]$ & 50 \\
\hline
\end{tabular}

\subsection{Based on the analytic power control strategy of HEB}

For the feature of HEB, the power analytic control strategy was build, which based on the power required of driver. In order to get the required torque of the HEB, driver give signals by electronic accelerator pedal or brake peda to distribute the torque from engine and motor and give consideration to the balance of the battery SOC by control strategy. Torque analysis for driver as show in fig 2

According to the layout and dynamic performance of HEB, when required torque is positive, the power management mode was established as show in fig 3. Engine optimal torque shows as Temax.Qemax, engine cutoff torque shows as Temin·Qemin , the engine launch speed shows as nlow.

The number describe as the work modes. When required torque is upper than Temax.Qemax, the work mode is the hybrid mode shows as number 1 ; When required torque between Temax.Qemax and Temin-Qemin. the work mode is engine work lonely mode shows as number 2; When the battery SOC is lower than its low limit and required torque is upper than Temin-Qemin, engine works in Temax.Qemax, an additional torque to charge the battery shows as number 3. When required torque is lower than Temin-Qemin and the battery SOC is lower than its low limit, engine supply drive torque and additional torque to charge the battery show as number 4 . When required torque is lower than Temin.Qemin and the battery SOC is lower than its low limit, motor drive HEB show as number 5 .

When required torque is negative or zero, according to battery SOC, whether recover the breaking energy. 


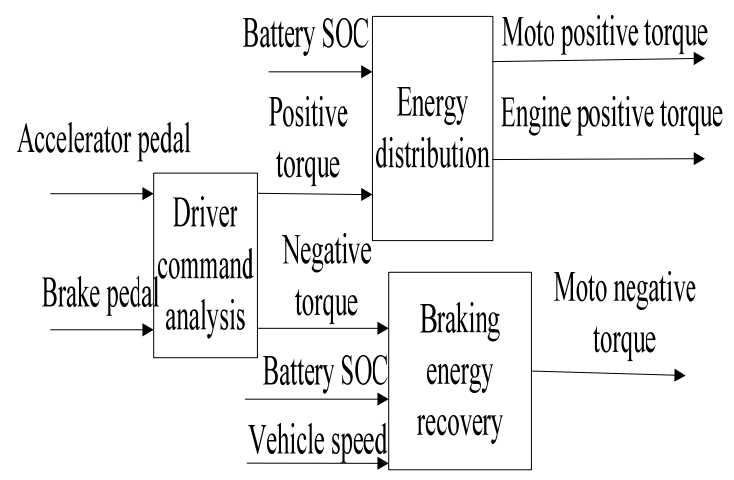

Fig. 2 . Energy management mode

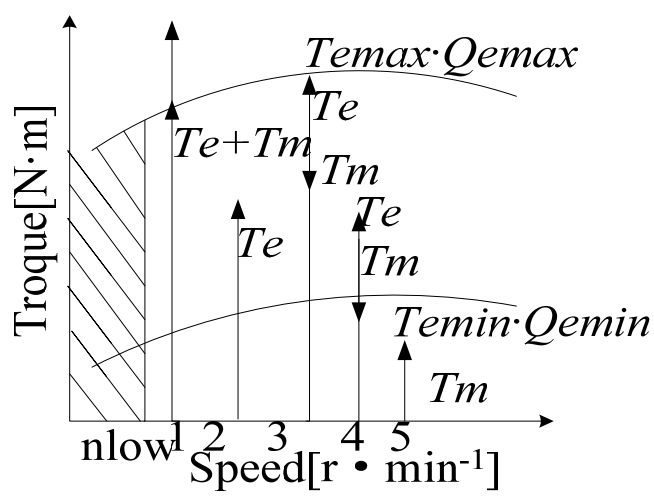

Fig. 3 . Torque analysis for driver

\section{Optimization of HEB control strategy}

The aim of optimization for HEB control strategy is to get to the best economy、 lowest emission and keep a long life for battery without sacrificing the dynamic performance, which is the goal Optimization of HEB control strategy. In essence, this leads to the solution of a constrained nonlinear programming problem whose expressions are as follow:

$$
\begin{cases}\min & F(x) \\ \text { s.t. } & g_{i}(x) \geq 0 \quad j=1,2, \cdots, m \\ & x_{j}^{\max } \leq x_{i} \leq x_{j}^{\min } \quad i=1,2, \cdots, n\end{cases}
$$

$F(x)$ is the objective function, $\left\{\mathrm{A}(\mathrm{x}) \backslash \mathrm{B}(\mathrm{x}) \cdots \mathrm{W}(\mathrm{x})\right.$ are the different funtion, $x_{j}$ the control strategy parameter. $x_{j}^{\max }$ and $x_{j}^{\min }$ are the control strategy parameter's upper value and lower value; $g_{i}(x)$ is the constraint condition.

\subsection{Objective function of HEB}

In this paper, optimization of HEB control strategy aims at the reduction of fuel consumption and preventing the battery from over charging or over discharging to keep the long life of the SOC while maintaining the driving performance .However, minimum fuel consumption and minimum fluctuation of battery SOC are conflicting in nature, so the objective function can be descriped as follows:

$$
\min \left\{\begin{array}{l}
F(x)=Q_{\text {fuel }}(x) \\
F(x)=\left|W_{\text {(SOCend - SOCini) }}(x)\right|
\end{array}\right.
$$

Where $Q_{\text {fuel }}(x)$ is the function of minimum fuel consumption; $\mid W($ soCend-SOCini) $\mid$ is minimum fluctuation of battery SOC. SOCend and SOCini is the final state value and initial state value.

\subsection{Control strategy parameters for optimization}

HEB control strategy is a complex, nonlinear question, which has much more different control parameters ,but it is almost impossible to optimize all of the parameters .In this paper, we choose these independent parameters, which have the most notable effect on the error probability and can be controlled directly are used as the design variables of the objective function as table 2 shows. C_SOC_hi means the up limit coefficient, C_SOC_hi means the lower limit coefficient, C_Trq_hi means engine optimal torque coefficient, $\mathrm{C}_{-}$Trq_low means engine cutoff torque coefficient.

Table 2. Pre-optimization control strategy parameters

$\begin{array}{lllll}\text { Variable name } & \text { C_SOC_hi } & \text { C_SOC_low } & \text { C_Trq hi } & \text { C_Trq } 10 w\end{array}$


$\begin{array}{lcccc}\text { Lower bound } & 0.5 & 0.05 & 0.5 & 0.05 \\ \text { Upper bound } & 1 & 0.5 & 1 & 0.5\end{array}$

\subsection{Constraint conditions for optimization}

The optimization of control strategy meets driving performance.but there are more and more driving performance parameters to be chosen for HEB. In this paper, the driving parameters of HEB are in accordance with prototype bus as described in table 3.

Table 3. Constraint conditions

\begin{tabular}{cl}
\hline Constraint & Description \\
\hline Maximum speed $[\mathrm{km} / \mathrm{h}]$ & $\geq 80$ \\
Maximum inclination & $\leq 12 \%$ \\
Acceleration time of $0 \sim 50 \mathrm{~km} / \mathrm{h}[\mathrm{s}]$ & $\leq 20$ \\
Chinese typical city bus absolute error[km/h] & $\leq 3$ \\
\hline
\end{tabular}

\section{HOA and optimization for HEB}

As an excellent global optimum algorithm, Genetic Algorithm has been applied widely in the optimization of HEV, but Genetic Algorithms get local optimization too easily and converge slowly. In order to get over the disadvantages of the slow convergence speed and stagnation behavior, a new hybrid optimization algorithm(HOA) that combine MIGA and NLPQL is proposed.

\subsection{Design of HOA}

MIGA $^{[10]}$ can divide the whole swarm into several sub-swarms, executes the operation of selection, cross over and mutation in a subpopulation, and periodically do immigration operation among the different islands. The pseudo-and some individual regular transfer from the different sub-swarms ,this transfer make MIGA higher effective 、 avoid premature convergence and apt to combine with other algorithms. But It also needs to spend much time to get optimization. As a local numerical optimum on method, NLPQL ${ }^{[1]}$ can solve non-linear、constrained optimize problems well, the algorithm combines NLPQL and genetic algorithm together, which makes genetic algorithm in the early stage play a powerful global search. It is easy to converge to the global optimum solution; in the later stage, NLPQL are used to deal with the overall situation of preoptimum solution, And it makes full use of NLPQL's latter part of the power of local search and eventually converges to the local optimal solution.

\subsection{Optimization for control strategy with HOA}

The HOA been integrated in ISIGHT software platform, which combined AVL-Cruise and MATLAB to optimize the control strategy of HEB. The sketch map of integration as show in fig 4. The optimized flow as followed:

(1)Initialization of the control strategy and parameter design

(2)The sub-swarm were randomly assigned, and make the generation evolution.

(3) Individual fitness should be calculated under the dynamic constraint. According to the order of probabilities $P_{c}$, the evolution was operated order crossover operation, and according to the order of probabilities $\mathrm{P}_{\mathrm{m}}$, the mutation operated.

(4)During the process of exploring the objective value in every iteration step, some individuals transfer from different islands to get the optimization.

(5)When MIGA finish, NLPQL locate the optimal areas to search the optimization result automatically. 


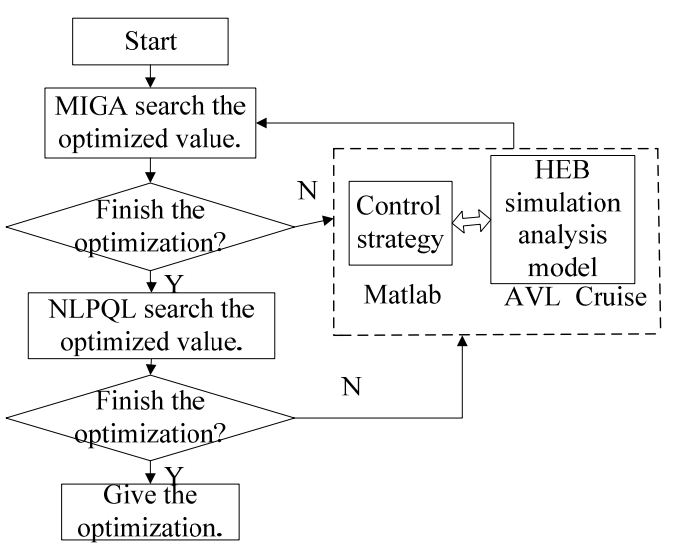

Fig. 4.The sketch map of integration

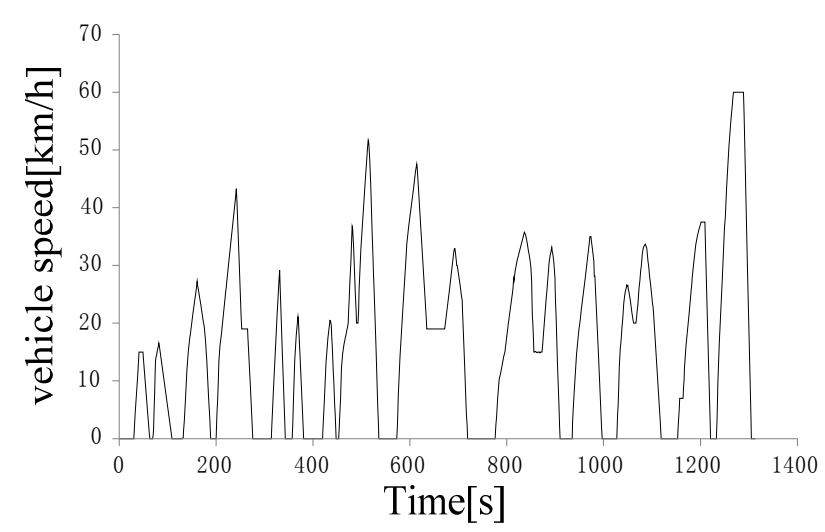

Fig. 5. CBC

\section{Simulation Results And Analysis}

In order to validate the validity of HOA, the vehicle simulation model is constructed in AVL Cruise, control strategy is constructed by MATLAB/Simulink. HOA is constructed by ISIGHT. Simulation works with typical city-bus driving-cycle in China (CBC) shows as fig 5. After 2000 generations evolved by MIGA alone , 1000 generations evolved by HOA, included 40 gradient optimization by NLPQL, and MIGA and HOA have the same numbers of island and the crossover and mutation rates The simulation optimal result used MIGA 、 HOA and non-optimal result of control strategy are listed in Table 4.

Table 4. Result of simulation

\begin{tabular}{cccc}
\hline Optimization variables and Objective & Non-optimal & MIGA & HOA \\
\hline C_Trq_hi & 0.8 & 0.92 & 0.99 \\
C_Trq_low & 0.3 & 0.28 & 0.26 \\
C_SOC_hi & 0.8 & 0.82 & 0.79 \\
C_SOC_low & 0.3 & 0.43 & 0.44 \\
Fuel consumption[L/100km ) & 31 & 28.23 & 27.98 \\
Power consumption[Kwh/100km] & 2.23 & 1.23 & -0.62 \\
Integrated fuel consumption[L/100km] & 31.69 & 28.59 & 27.78 \\
\hline
\end{tabular}

Optimal procedures for fuel consumption and power consumption with HOA as show in fig 6.in the early evolution stages, it searches more quickly and efficiently in the whole global optimization. After the evolution of four hundred generations, it converges to the global optimization .but some evolved individuals escape from local optimal area to explore the global areas for overcoming the premature. At the last time of optimal procedures, NLPQL locate the optimum areas automatically, which eventually converges to the optimal solution by means of the gradient technique quickly and can't explore global optimum areas.
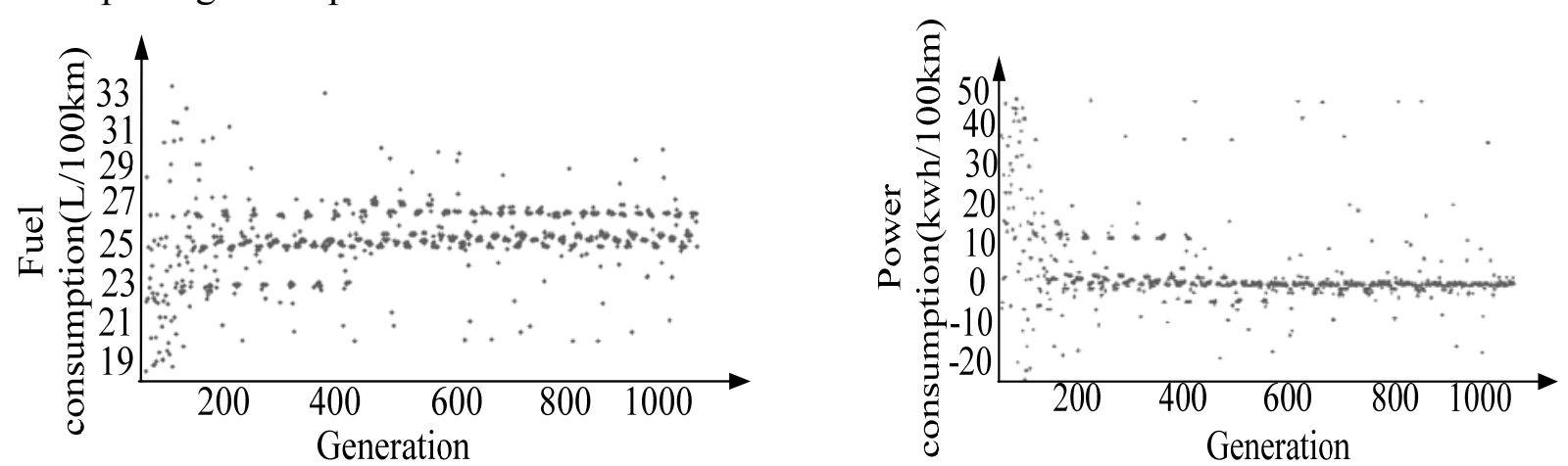

Fig. 6. Optimal procedures for fuel consumption and power consumption

Compared with the case of non-optimization, the Hundred-kilometer power consumption is reduced from $2.23(\mathrm{kwh} / 100 \mathrm{~km})$ to $-0.62(\mathrm{kwh} / 100 \mathrm{~km})$, battery SOC maintains balance basically. 
The hundred-kilometer fuel consumption reduces by $10 \%$, integrated fuel consumption reduced by $12 \%$. Compared with MIGA and HOA, Simulation results show that it's no advantage for the fuel consumption and power consumption basically. But it take 24 hours to get the optimum solution by MIGA and 12 hours to get the global optimization by HOA, it greatly enhanced search efficiency compared with MIGA.

Relationship between control strategy parameter and objective function shows as fig 7. In order to keep the balance of the battery SOC, fuel consumption should keep from $27.5(\mathrm{~L} / 100 \mathrm{~km})$ to $28.4(\mathrm{~L} / 100 \mathrm{~km})$; the engine optimal torque coefficient keep from 0.88 to 0.99 ; the engine cutoff torque coefficient keep from 0.2 to 0.27 ; the battery SOC low limit coefficient keep from 0.2 to 0.27 and the battery SOC up limit coefficient should keep from 0.75 to 0.82 .the result shows that fuel consumption and power consumption is a pair of a mutually conflicting objective functions, the balance of the battery SOC and reducing the fuel consumption depend on multiple control strategy parameters. These results can provide important design guidance of control strategy for hardwarein-loop test.
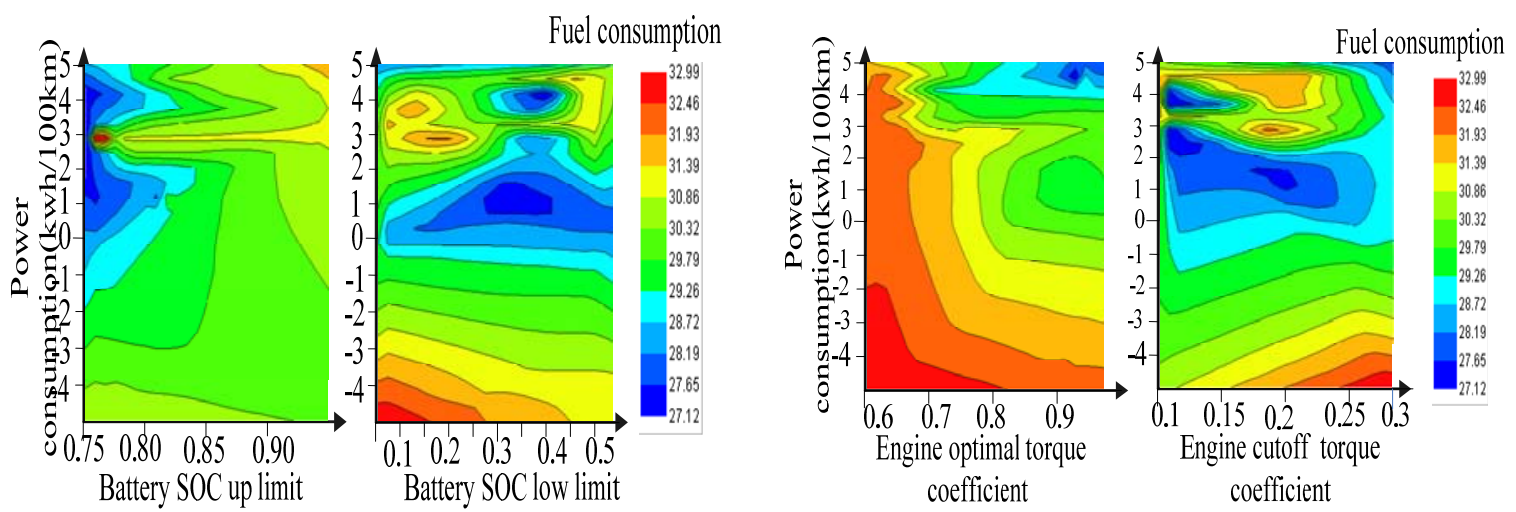

\section{Conclusion}

Fig.7. Relationship between control strategy parameter and objective function

The HOA been integrated in ISIGHT software platform, which combined AVL-Cruise and MATLAB to optimal the control strategy of HEB. Compared with using manual programming, it takes little time to design HOA.The simulation result shows that:

(1)The calculation results show that HOA improve fuel economy and keep the balance of the battery SOC when the automobile remains its original dynamics. Hundred-kilometer integrated fuel consumption is reduced by $12 \%$ and kept the balance of the battery SOC by -0.67 .

(2)Compared with MIGA, HOA can get global optimal solution and reduce search time, optimization time was shortened by 12 hours.

(3)Rule between control strategy parameters and objective function is obtained, which give valuable guidance for hardware-in-loop test.

\section{Acknowledgment}

The work is supported by State 863 Theme Projects during the Twelfth Five-year Plan Period (No.2012AA111603) and Graduate innovation grant of Henan University of Science \& Technology (CXJJ-YJS-Z004)

\section{References}

[1] Gao Jianping, Zhu G M G,Strangas E G, et al, Equivalent Fuel Consumption Optimal Control of a Series Hybrid Electric Vehicle [J].Journal of Automobile Engineering, 2009,223(8):1003-1018.

[2] M.Ehsan, Y.Gao, and A.Emadi, Modern Electric, Hybrid Electric and Fuel Cell Vehicles: Fundamentals, Theory, and Design, 2nd ed .New York:Taylor \&Francis,2010. 
[3] Yue Shubiao, Gao Jianping, Yang Luhang, Start/Stop Control Strategy for Parallel Hybrid Electric Vehicle , [J] Journal of Henan University of Science \&Technology(Natural Science) 2011, 32(2): 21-26.

[4] Piccolo A, Ippolito L,Galdi V, Optimization of energy flow management in Hybrid Electric Vehicles via Genetic Algorithms[C] // Conferentce on Advanced Intelligent Mechatronics. Como: IEEE/ASME Int, 2001.

[5] Huang B,Wang Z,Xu Y. Multi-objective genetic algorithm for hybrid electric vehicle parameter optimization $[\mathrm{C}] / /$ Proceeding IEEE International Conferrence on Intelligent Robots and Systems, Washington: IEEE, 2006: 5177 - 5182.

[6] S.M.MeMi Ansarey M, Mohsen Mohammadian and S.M.Taghi Bathaee, Power Flow Distribution For Hybrid Fuel Cell Vehicle Via Genetic Algorithm Method, SAE. 2004_0 1-3040A. Sakai. Y Sasaki A. Otomo E Kawahata A, Matui. Toyota Braking System for Hybrid Vehicle with Regenerative System.

[7] HASANZADEH A,ASAEI B,EMADI A. Optimum design of series hybrid electric buses by genetic algorithm [C]//IEEE ISIE, Dubrovnik,Croatia,2005.

[8] SCHOEGGL P, KRIEGLER W. Virtual optimization of vehicle and powertrain parameters with consideration of human factors[R]. SAE Paper 2005-01-1945.

[9] Zhang Xin, Song Jianfeng, Tian Yi, Multi - objective Optimization of Hybrid Electric Vehicle Control Strategy with Genetic Algorithm [J].Journal of Mechanical Engineering, 2009,45(2).

[10]Seo, Janghoo;Ooka, Ryozo;Kayo, Genku , Study on optimal energy system design for apartment house using genetic algorithms [C]//International Building Performance Simulation Association 2009 ,11th International IBPSA Conference p 1830-1834, 2009.

[11]Liu Guoqing, Chen Jie, Na Tianming, NLPQL of control rules for improving fuel economy of a parallel hydraulic hybrid bus, International Journal of Modelling, Identification and Control, v 7, n 4, p 315-320, 2009. 